\title{
A Systematic Review of Studies on Blood Pressure in Iranian Pediatric Population: First Report From the Middle East and North Africa
}

\author{
Zahra Fallah, ${ }^{1,2}$ Shirin Djalalinia, ${ }^{3,45}$ Mostafa Qorbani, ${ }^{6}$ Farshad Farzadfar, ${ }^{3,4}$ and Roya \\ Kelishadi $1^{1,}$ \\ ${ }^{1}$ Department of Pediatrics, Child Growth and Development Research Center, Research Institute for Primordial Prevention of Non-Communicable Disease, Isfahan University of \\ Medical Sciences, Isfahan, IR Iran \\ ${ }^{2}$ Student Research Center, Isfahan University of Medical Sciences, Isfahan, IR Iran \\ ${ }^{3}$ Non Communicable Diseases Research Center, Endocrinology and Metabolism Population Sciences Institute, Tehran University of Medical Sciences, Tehran, IR Iran \\ 4 Endocrinology and Metabolism Research Center, Endocrinology and Metabolism Research Institute, Tehran University of Medical Sciences, Tehran, IR Iran \\ 5 Developmental Research and Technology Center, Deputy of Research and Technology, Ministry of Health and Medical Education, Tehran, IR Iran \\ ${ }^{6}$ Department of Community Medicine, School of Medicine, Alborz University of Medical Sciences, Karaj, IR Iran \\ ${ }^{*}$ Corresponding author: Roya Kelishadi, Department of Pediatrics, Child Growth and Development Research Center, Research Institute for Primordial Prevention of Non-Communicable \\ Disease, Isfahan University of Medical Sciences, P. O. Box: 81676-36954, Isfahan, IR Iran. Tel: +98-3136691216, Fax: +98-3136687898, E-mail: kelishadi@med.mui.ac.ir
}

Received 2015 October 28; Accepted 2015 November 12.

\begin{abstract}
Context: Blood pressure (BP) tracks from childhood to adulthood, and has ethnic variations. Therefore, it is important to assess the situation of pediatric BP in different populations. This study aims to systematically review the studies conducted on BP in Iranian children and adolescents.

Evidence Acquisition: We conducted a systematic review on published and national data about pediatric BP in Iran, our search was conducted in Pub Med, Medline, ISI, and Scopus, as well as in national databases including Scientific Information database(SID), IranMedex and Irandoc from 1990 to 2014.

Results: We found 1373 records in the primary search including 840 from international and 533 from national databases. After selection and quality assessment phases, data were extracted from 36 papers and four national data sources. Mean systolic BP(SBP) varied from 90.1 $\pm 14 \mathrm{mmHg}(95 \% \mathrm{CI} 89.25,90.94)$ to $120.2 \pm 12.3(118.98,121.41) \mathrm{mmHg}$, and for diastolic BP(DBP) from $50.7 \pm 11.4(50.01,51.38)$ to $79.2 \pm 12.3$ (77.95, $80.44) \mathrm{mmHg}$. The frequency of elevated BP had large variation in sub-national studies with rates as low as $0.4 \%(0.009,1.98)$ for high SBP and as high as $24.1 \%(20.8,27.67)$ for high DBP. At national level, three surveys reported slightly raised rates of elevated BP from 2009 to 2012. Conclusions: The findings provide practical information on BP levels in Iranian pediatric population. Although differences exist on the findings of various studies, this review underscores the necessity of tracking BP from childhood, and implementing interventions for primordial prevention of hypertension.
\end{abstract}

Keywords: Non-Communicable Disease, Blood Pressure, Child, Adolescent, Iran, Burden

\section{Context}

In the last decades and almost all around the world, the disease burden profile has changed from communicable diseases in childhood to non-communicable diseases (NCDs) in adulthood $(1,2)$. Meanwhile, the worldwide healthy adjusted life expectancy (HALE) has grown slower than life expectancy ( 0.8 years for each 1 year) and in a few countries HALE even has been reduced $(3,4)$. In addition, non-fatal health outcomes have resulted in increasing years lived with disability (YLD), worldwide (5). The Global Burden of Diseases (GBD) study on 2010 identified high blood pressure (BP) as the top global burden risk factor. In most parts of Asia, Middle East, North Africa, and Central Europe, high BP was the leading factor. It had worldwide attributable deaths of about 9.4 million and disability adjusted life years (DALYs) of about 173.6 million and comprised 53 percent of ischemic heart disease (IHD) DALYs (1). Moreover, this study defined IHD, lower respiratory infections, and stroke as the three major causes for years of life lost (YLL) due to premature death (6), with high BP as one of the most important risk factors for all of them.

In 2001, high BP resulted in 7.6 million premature deaths and 92 million DALYs; it also caused 54\% of strokes and $47 \%$ of IHDs worldwide (7). In 2002, IHD and stroke were the two principal causes of global death (8). The world health organization (WHO) ranked high BP (13\% of global death) (9) and, cardiovascular diseases (CVDs) (45\% of global deaths) (10) as the leading risk factors for global

Copyright (C) 2016, Growth \& Development Research Center. This is an open-access article distributed under the terms of the Creative Commons Attribution-NonCommercial 4.0 International License (http://creativecommons.org/licenses/by-nc/4.0/) which permits copy and redistribute the material just in noncommercial usages, provided the original work is properly cited. 
death in 2009 and 2011, respectively.

It is well documented that elevated BP during adulthood roots in childhood (11-13). Given the importance of tracking BP from childhood, the Fourth report on the diagnosis, evaluation, and treatment of high BP in children and adolescents highlighted that all children aged above 3 years, who are seen in a medical setting, should have their BP measured (14). The growing pattern of NCDs and the pattern of the GBD are of special concern in low- and middle-income countries (1). As a low-middle income country located in the Middle East and North Africa (MENA) region, Iran is facing rapid epidemiological transition and change in the disease pattern (15). Therefore, increasing our knowledge on the risk factors of NCDs, would be helpful for designing and implementing timely preventive health programs. There are scattered and independent studies in this regard in Iran. Their results mostly are from limited populations or confined areas. It seems necessary to cumulate their findings and estimate the overall burden of elevated BP for the country. Furthermore, comparing data at the local and subnational levels might yield useful results, which could be used by health policy makers and health workers. None of the published articles has considered this issue. Even the GBD study has drawn an overview of the situation at global level and does not provide each country's specific situation. Therefore in the current review, first we looked for the studies conducted on BP among Iranian pediatric population. Thereafter, we tried to deliberate the relevant trends and burdens in the country. Due to the scarcity of accessible information in many areas of the country, we need to use advanced statistical modeling methods in order to impute the missed data $(16,17)$.

\section{Evidence Acquisition}

The present systematic review is a sub-component of cardio-metabolic risk factors burden study in Iranian children and adolescents which itself is a part of the National and Sub-national burden of diseases, injuries and risk factors (NASBOD) study in Iran. We have described detailed designs and protocols elsewhere $(18,19)$, and here we explain it in brief.

\subsection{Outcome Definition}

The major outcome in the current review was elevated BP. According to the US national heart, lung, and blood institute (NHLBI), BP is considered normal when the systolic and diastolic values are less than the $90^{\text {th }}$ percentile for the child's age, sex, and height. Prehypertension, is diagnosed when a child's average BP is above the 90th percentile but below the 95th. Every adolescent with a BP greater than $120.80 \mathrm{mmHg}$ is also diagnosed to have prehypertension, even if the BP would be below the $90^{\text {th }}$ percentile. Stage I hypertension is diagnosed if a child's BP is greater than the 95th percentile but less than or equal to the 99th percentile plus $5 \mathrm{mmHg}$. Stage II hypertension is diagnosed if a child's BP is greater than the 99th percentile plus $5 \mathrm{mmHg}(14)$. In the current review, the majority of the retrieved articles had used these cut points or earlier definitions proposed by NHLBI $(20,21)$. Other definitions have been used in some of them $(22,23)$.

\subsection{Measures Definition}

Measures consisted of the values/mean(Standard Deviation, SD) values of SBP and/or DBP, or the frequency rates of high BP. In all studies selected for this review, BP value was measured by sphygmomanometer through brachial artery with appropriate cuff size and was reported in $\mathrm{mmHg}$ or $\mathrm{cmHg}$. In most of them, BP was measured two to three times in a single session and the mean value was recorded. In some others, two to three measurements were made on separate occasions and the last one was included in their analysis.

\subsection{Search Strategy}

We searched international databases including PubMed/Medline, ISI web of Science, and Scopus, as well as the Iranian scientific databases (IranMedex, Scientific Information Database (SID), Irandoc) from January 1990 to January 2014.

The search terms were as follows: hypertension, blood pressure, high blood pressure, systolic pressure, diastolic pressure, arterial pressure in any possible combination with school OR student OR girl OR boy OR child* OR adolescen* OR pediatr* OR paediatr* AND Iran for searching in the International databases; the Farsi equivalent of these terms were used for searching the national databases. A definite search strategy for each database was also designed and used (Appendix 1). Both search methods results were considered. We also looked at the reference list of relevant retrieved articles to find more publications.

\subsubsection{National Data}

To the best of our knowledge the only nationwide study on cardio-metabolic risk factors in Iranian pediatric population with the age specific definitions are the surveys of a national surveillance program entitled: Childhood and adolescence surveillance and prevention of adult non-communicable diseases (CASPIAN) study $(24,25)$. It has been conducted in four different surveys from 2003 to 2012 (25-29). We used the source data of these surveys.

\subsection{Inclusion and Exclusion Criteria}

We included all cross sectional studies on BP values and prevalence of high BP in 6 - 18 year-old Iranian population. We also included cohort and case-control studies if they had reported their baseline data. We limited the 
Fallah Z et al.

search to human studies conducted from January 1990 to January 2014 in Iran (Iran, I.R.Iran, Islamic Republic of Iran) or with Iran in the affiliation of the authors with no restriction in the language. Non-human studies and redundant publications were excluded. All document types were included at the first stage. We excluded the studies that reported population normal values or percentiles exclusively or reported findings in more extended age groups than our study age range, or did not use pediatric specific definitions for BP classification. In the case of finding multiple publications from one study, we selected the more comprehensive one.

\subsection{Data Management}

All retrieved publications underwent selection and qualification processes. The search was repeated throughout the study period to add any newly published articles. The selected documents were saved in computer files with back-ups.

\subsection{Selection Process}

In the first stage, the retrieved titles were screened to find relevant articles, at the second stage the abstracts were screened, and at the third stage full texts of relevant papers were screened (Appendix 2).

\subsection{Quality Assessment}

For quality assessment, we considered our study eligibility criteria, study design, sample size, sampling method, response rates, measurement tools and their calibration, as well as measurement methods and estimates. Two independent reviewers (ZF and RK) qualified the articles and the poor rated ones were excluded.

\subsection{Data Extraction}

The data extraction process is explained in detail elsewhere (19). For most papers, confidence intervals (CI) were calculated. For six papers for which complementary data were needed, we tried to make contact with main authors but unfortunately the response rate was not favorable.

\section{Results}

The search algorithm including the number of initial search results and included studies are shown in Appendix 2. At first step, we found 840 articles in international and 533 articles in domestic databases. After three steps of selection and then qualifying processes, we included 36 articles (30-68) (Tables 1 and 2) in our systematic review.

Altogether, this review included 1,096,263 total population (515,098 girls, 47\%) and 277 data-points. They were from four national, one provincial, 29 district and two community study levels.
Mean BP value had variation in different studies. The least value of mean SBP $(90.10 \pm 4.00 \mathrm{mmHg}(95 \% \mathrm{CI}$ $89.25,90.94)$ ) and mean DBP (50.7 \pm 11.40 (50.01, 51.38) $\mathrm{mmHg}$ ) are both reported from Tehran city in the same study (54). The highest mean values reported are $120.2 \pm$ $12.30(118.98,121.41) \mathrm{mmHg}$ for mean SBP from Kerman city (59) and 79.20 $\pm 12.30(77.95,80.44) \mathrm{mmHg}$ for mean DBP from Isfahan city (53). The study of Kerman city also had the second rank of highest DBP, i.e. $76 \pm 11.4(4.87,77.12)$ mmHg (59).

As presented in Tables 1 and 2, almost all articles reported mean BP values. From 36 published articles, fifteen reported prevalence rates of isolated high SBP and high DBP (Table 1). Fifteen articles reported the prevalence of high SBP and/or high DBP (Table 2).

Table 1 also presents the national data of CASPIAN studies (66-68). The reported mean BP values do not show large variations in these three surveys. The CASPIAN-I study was a nationwide survey conducted in 2003 2004 in 23 provinces, and included 21,111 students, aged 6 - 18 years. Elevated BP was defined as values equal or greater than 95th percentile, and the prevalence of high SBP, DBP as well as SBP and/or DBP in total population was $4.2 \%(3.96,4.51), 5.4 \%(5.05,5.67)$ and $7.7 \%$ (7.38, 8.11), respectively (66). The CASPIAN-III study was conducted in 2009 - 2010 among 5,738 students aged 10 - 18 years living in 27 provinces. It reported prevalence rates for high $\mathrm{BP}$ (values equal or greater than 90 th percentile) as follows: $3.8 \%(3.31,4.32), 3.3 \%(2.86,3.8)$, and $6 \%(5.41,6.65)(66)$. The corresponding figures reported by CASPIAN-IV study, conducted in 2011 - 2012 on 13,486 students from 30 provinces, were $4.17 \%$ (3.84, 4.52), $4.33 \%(3.99,4.68)$ and $6.88 \%(6.45,7.32)$, respectively (68). These rates were slightly higher than those reported from CASPIAN-III study.

As it is obvious from these two tables, the reported range of high BP in Iranian pediatric population in subnational studies in the two past decades varies widely, with rates as low as $0.40 \%(95 \%$ CI $0.009,1.98)$ for high SBP in Tehran province for 15 - 19-year-old boys (39-41) and as high as $24.10 \%(20.80,27.67)$ for high DBP in KhorasanRazavi province (46). Moreover, in national screening of 6-year-old children at school entry, the prevalence of high BP was 0.10\% (0.09, 0.109) (31).

Based on the use of $\geq 95$ th percentile to define hypertension, the prevalence of hypertension would be expected to be around 5\% and in fact 1 - 3\% (69). Our findings showed that the number of sub-national studies in Iranian pediatric population that reported high BP rates above $5 \%(30,45,46,55,58,61,62,65)$ was equal to studies that reported rates below $5 \%(31,34,36,42,44,48,49,64)$. In eight papers, the rates from both ranges (below and above 5\%) for different age or sex categories are reported (37-41, 43, 50, 57, 63). Seven studies reported at least one prevalence rate of elevated BP of more than 10 percent $(30,37-39,41,43,50,62,63)$. 
Fallah Z et al.

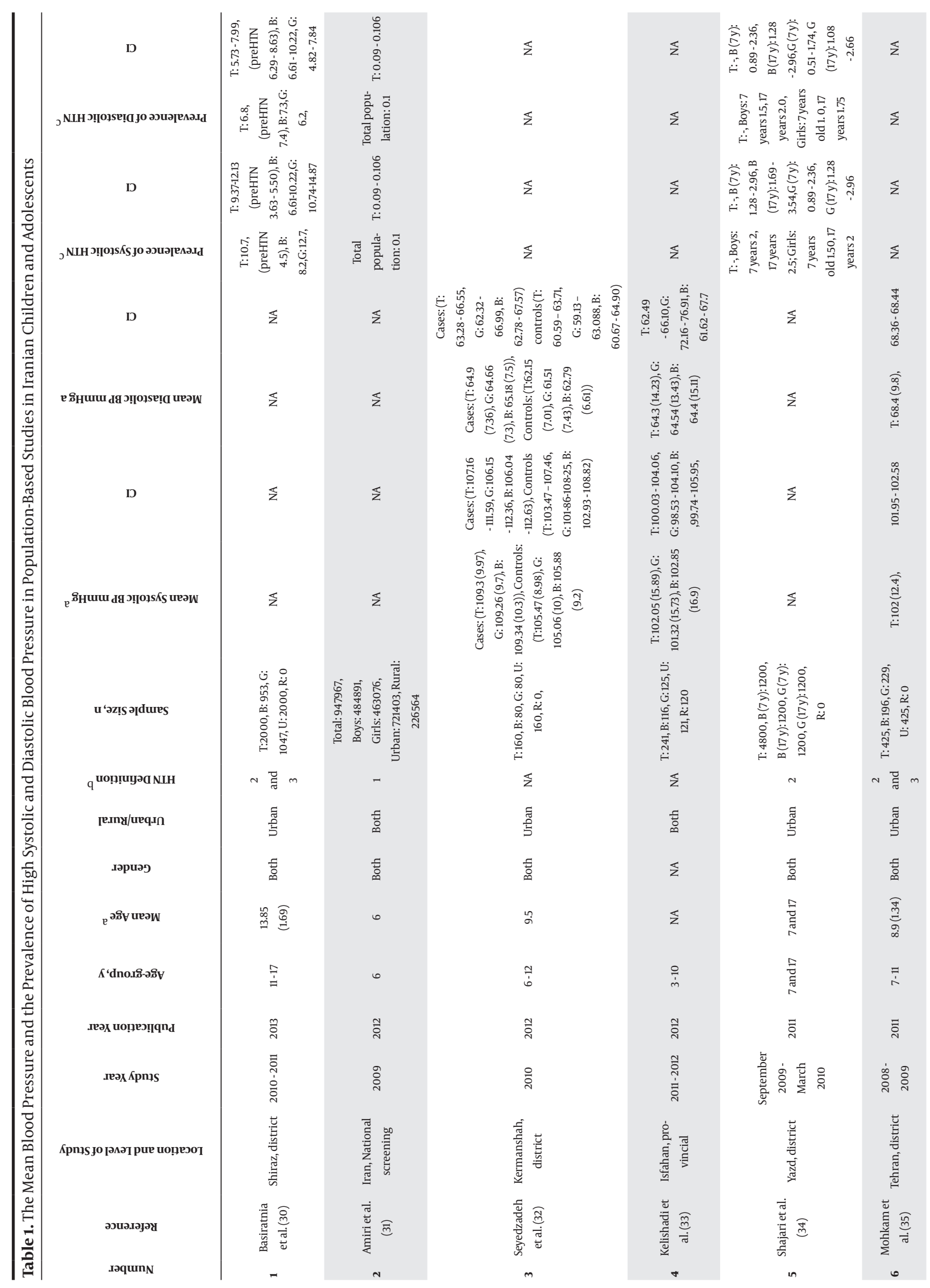


Fallah Z et al.

\begin{tabular}{|c|c|c|c|c|c|}
\hline$\frac{\pi}{z}$ & 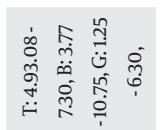 & 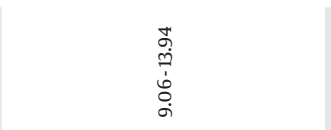 & $\begin{array}{l}\text { Fे } \\
\dot{j} \\
\text { j̇ }\end{array}$ & 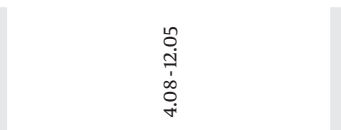 & $\begin{array}{l}\stackrel{m}{\infty} \\
\stackrel{\tilde{O}}{0} \\
0\end{array}$ \\
\hline$\Sigma$ & 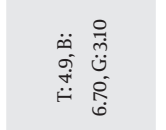 & $\stackrel{\cong}{\rightrightarrows}$ & $\underset{+}{\circ}$ & $\stackrel{\leftrightarrow}{N}$ & $\stackrel{\infty}{\oplus}$ \\
\hline $\bar{z}$ & 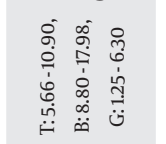 & 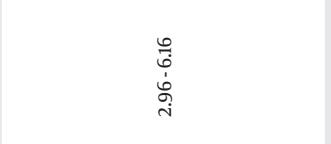 & $\begin{array}{l}\vec{\sigma} \\
\dot{+} \\
\dot{b} \\
\stackrel{b}{h}\end{array}$ & $\begin{array}{l}\text { ñ } \\
\text { ஸे } \\
\stackrel{\tilde{y}}{0}\end{array}$ & $\begin{array}{l}\vec{n} \\
\stackrel{1}{0} \\
\stackrel{\Delta}{0}\end{array}$ \\
\hline$\S$ & 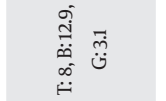 & F & $\ddot{n}$ & $\exists$ & วิ \\
\hline $\begin{array}{l}\text { q. } \\
\dot{0} \\
\dot{0} \\
\dot{0}\end{array}$ & 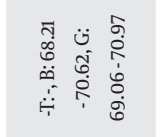 & 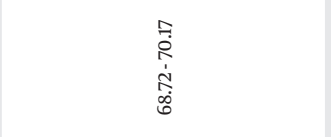 & 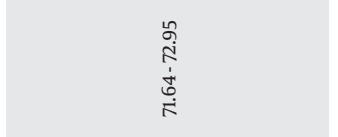 & 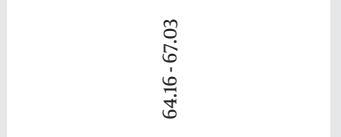 & 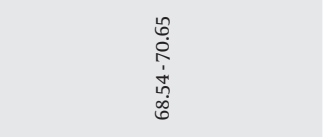 \\
\hline 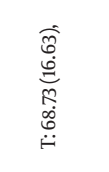 & 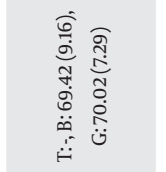 & 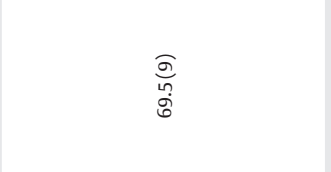 & $\frac{\sqrt{0}}{\pi}$ & 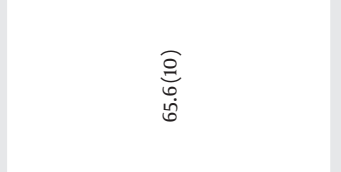 & ōo \\
\hline 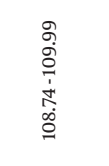 & 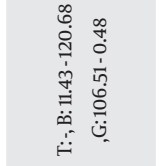 & 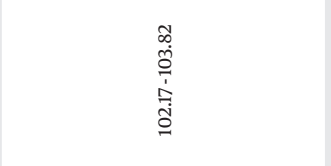 & $\begin{array}{l}\stackrel{\circ}{\circ} \\
\stackrel{\oplus}{=} \\
\stackrel{0}{0} \\
\stackrel{0}{0}\end{array}$ & 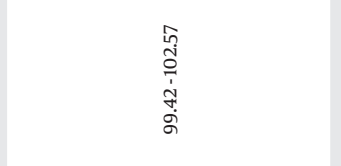 & 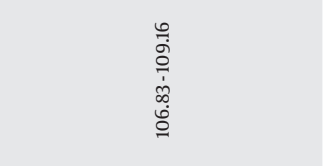 \\
\hline 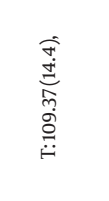 & 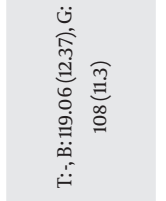 & $\underset{\underline{m}}{\Xi}$ & 징 & $\stackrel{\Xi}{\Xi}$ & $\begin{array}{l}\Xi \\
\stackrel{\Xi}{\varrho} \\
\stackrel{\infty}{G}\end{array}$ \\
\hline 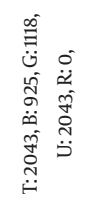 & 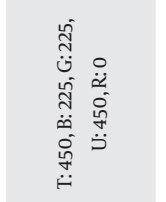 & $\begin{array}{l}\stackrel{\infty}{\circ} \\
\stackrel{\leftrightarrow}{\infty}\end{array}$ & 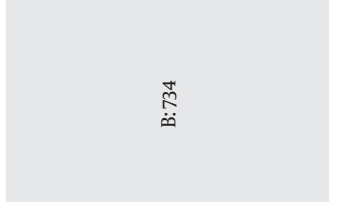 & 兽 & 恖 \\
\hline$r$ & $a$ & $\infty$ & $\infty$ & $\infty$ & $\infty$ \\
\hline $\begin{array}{l}\text { 퓸 } \\
\text { 吉 }\end{array}$ & 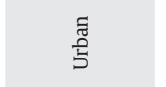 & 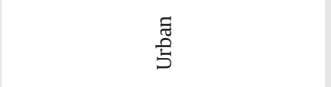 & $\begin{array}{l}\text { 歌 } \\
\text { 咅 }\end{array}$ & 超 & 駕 \\
\hline 咅 & 言 & $\frac{\pi}{\sum \pi}$ & $\frac{\frac{\pi}{\pi}}{2}$ & $\frac{\frac{0}{\pi}}{\Sigma}$ & $\frac{\frac{\varrho}{\tilde{m}}}{\sum}$ \\
\hline 豆需 & $\begin{array}{l}\widehat{\mathbb{E}} \\
\stackrel{0}{0} \\
\underline{0}\end{array}$ & $\underset{\Xi}{\Xi}$ & $\underset{\substack{\Xi \\
\stackrel{\infty}{0} \\
\stackrel{\infty}{0}}}{ }$ & 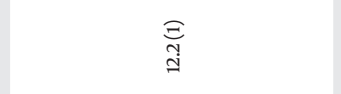 & $\stackrel{\Xi}{E}$ \\
\hline : & $\stackrel{\vec{b}}{\dot{m}}$ & $\stackrel{\frac{\pi}{3}}{\stackrel{5}{\circ}}$ & $\stackrel{9}{\dot{\varphi}}$ & $\stackrel{⿱ 亠 t}{\dot{0}}$ & $\stackrel{9}{\dot{m}}$ \\
\hline$\overline{\bar{~}}$ & స్ & $\overline{\bar{\sim}}$ & 产 & 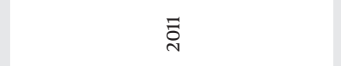 & స్ \\
\hline 它 & :े & 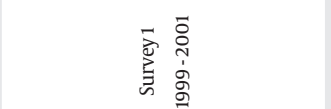 & 氛 & 离悹 & 空 今ั่ \\
\hline 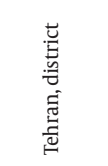 & 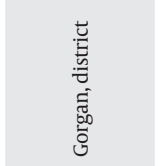 & 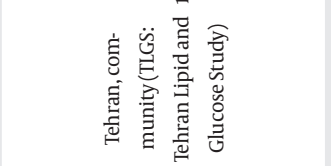 & 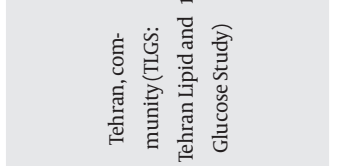 & 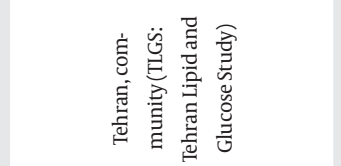 & 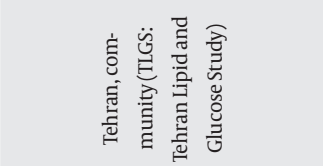 \\
\hline 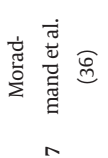 & 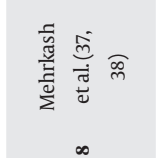 & 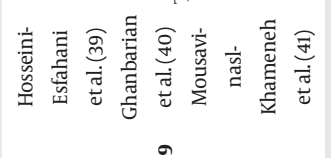 & 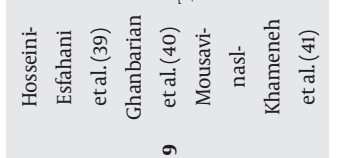 & 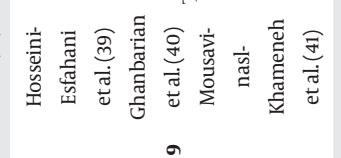 & 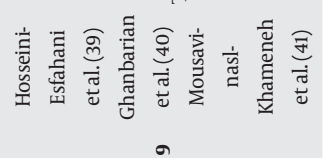 \\
\hline
\end{tabular}


Fallah Z et al.

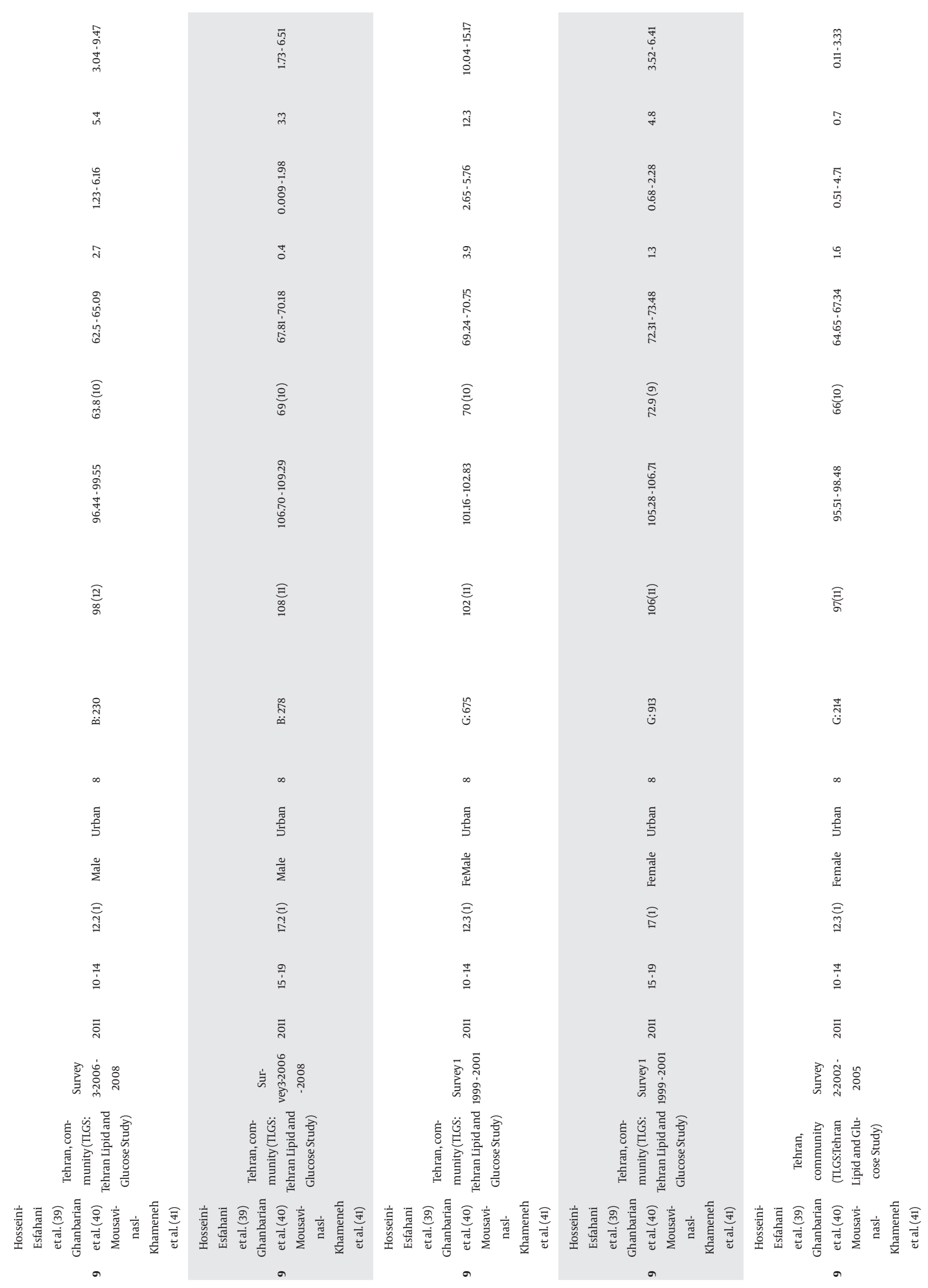




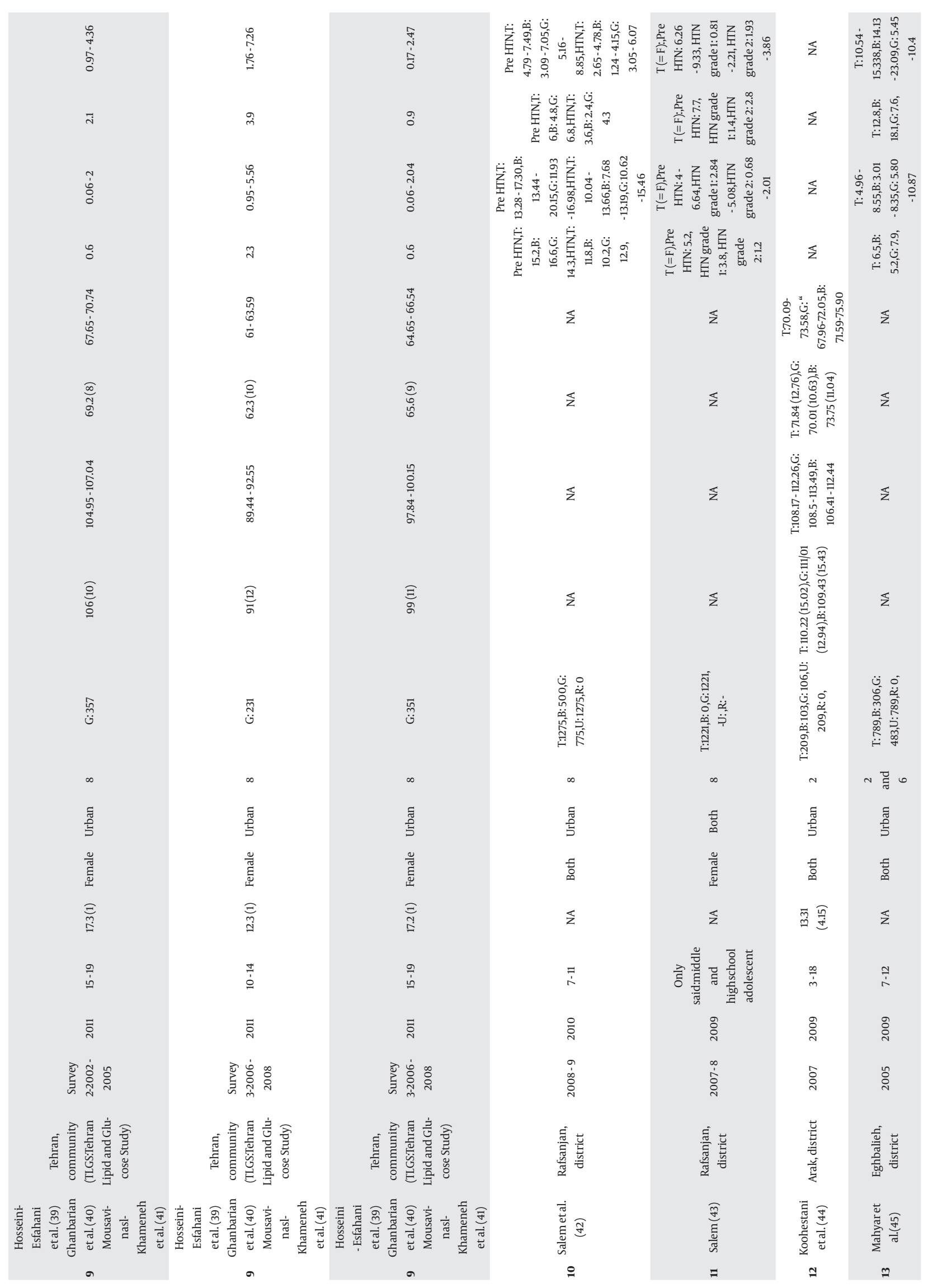




\begin{tabular}{|c|c|c|c|c|c|c|c|c|c|c|c|}
\hline 噢 & 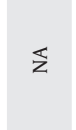 & $\bar{z}$ & 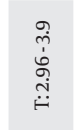 & 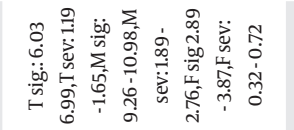 & $\bar{z}$ & 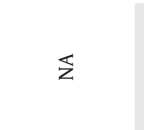 & $\Sigma$ & $\bar{z}$ & $\Sigma$ & $\Sigma$ & $\Sigma$ \\
\hline 苞 & $\bar{z}$ & $\Sigma$ & $\stackrel{\check{n}}{\leftrightarrow}$ & 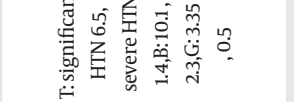 & $\Sigma$ & $\tilde{z}$ & $\underline{\xi}$ & $\Sigma$ & $\Sigma$ & $\bar{z}$ & 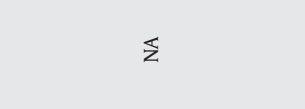 \\
\hline 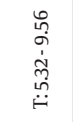 & $\bar{z}$ & $\bar{z}$ & 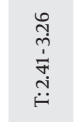 & 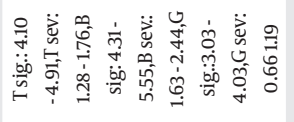 & $\Sigma$ & $\widehat{z}$ & $\S$ & $\tilde{z}$ & $\Sigma$ & $\Sigma$ & $\S$ \\
\hline 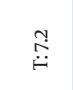 & $\bar{z}$ & $\bar{z}$ & 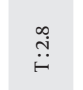 & 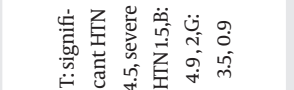 & $\bar{z}$ & $\S$ & 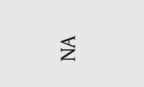 & 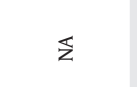 & $\Sigma$ & 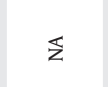 & $\S$ \\
\hline 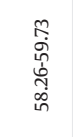 & 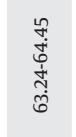 & 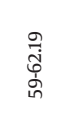 & 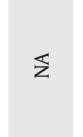 & $\Sigma$ & 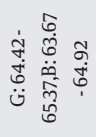 & 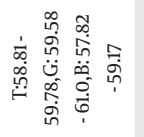 & 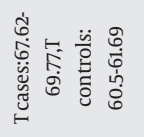 & 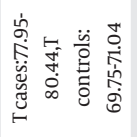 & 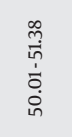 & 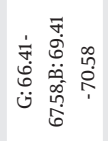 & 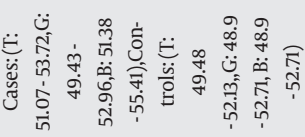 \\
\hline 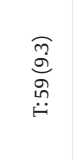 & 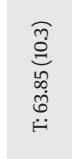 & 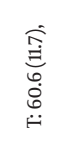 & $\bar{z}$ & $\widehat{z}$ & 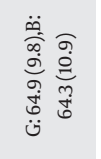 & 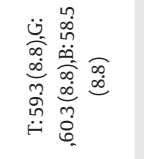 & 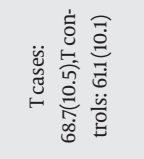 & 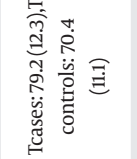 & 俤 & 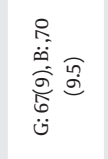 & 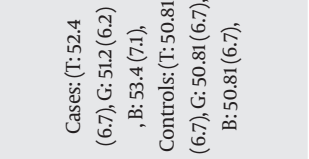 \\
\hline 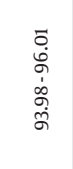 & 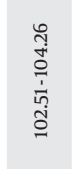 & 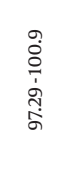 & $\bar{z}$ & $\Sigma$ & 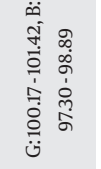 & 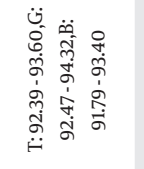 & 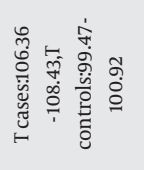 & 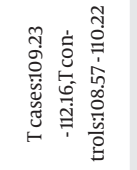 & 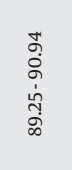 & 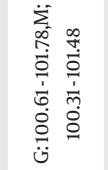 & 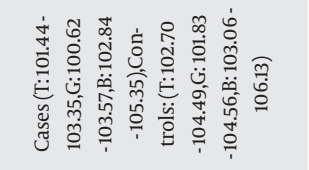 \\
\hline 兹 & 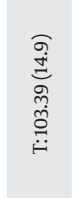 & 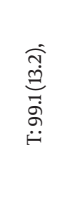 & $\frac{\pi}{z}$ & $\hat{z}$ & 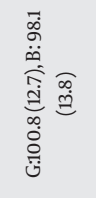 & 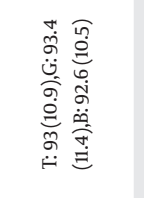 & 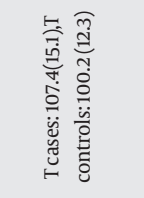 & 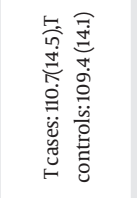 & 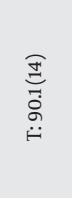 & 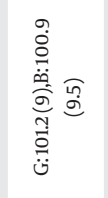 & 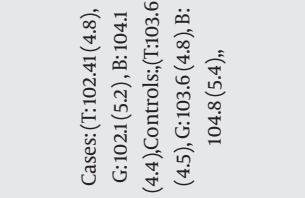 \\
\hline 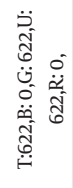 & 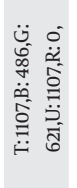 & 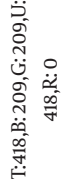 & 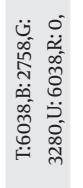 & 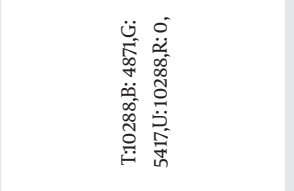 & 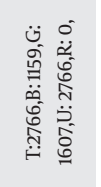 & 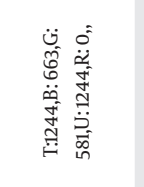 & 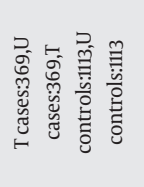 & 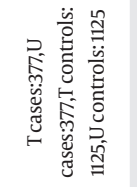 & 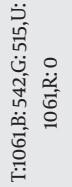 & 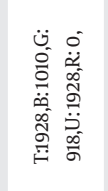 & 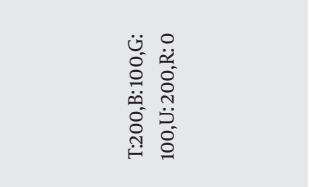 \\
\hline$a$ & . & $\sim$ & N & $\sigma$ & N & $\Sigma$ & $\sim$ & $\sim$ & . & $\sim$ & 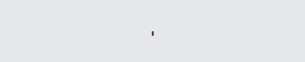 \\
\hline 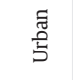 & 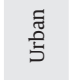 & 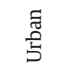 & 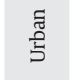 & $\begin{array}{l}\text { 듊 } \\
\text { ड़ }\end{array}$ & 胥 & 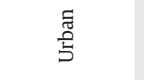 & 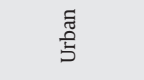 & 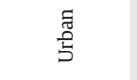 & 䔍 & $\begin{array}{l}\text { हूँ๊ } \\
\text { 馬 }\end{array}$ & 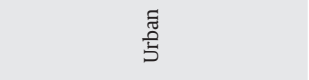 \\
\hline $\begin{array}{l}\text { 节 } \\
\text { 离 }\end{array}$ & 吉 & 营 & 总 & 咅 & 总 & 吉 & 营 & 咅 & 咅 & 总 & 咅 \\
\hline 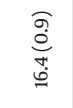 & 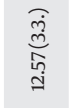 & 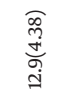 & $\S$ & $\Sigma$ & 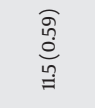 & $\Sigma$ & . & & ' & ' & 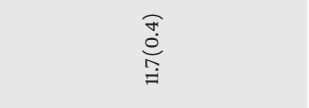 \\
\hline$\stackrel{\vec{D}}{\stackrel{1}{n}}$ & $\frac{\infty}{\dot{b}}$ & $\stackrel{\infty}{\dot{m}}$ & $\stackrel{\infty}{\stackrel{\oplus}{\oplus}}$ & $\stackrel{\eta}{\dot{b}}$ & 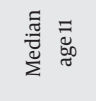 & $\stackrel{\overbrace{}}{0}$ & 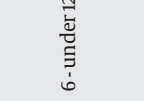 & $\stackrel{\infty}{\beth}$ & $\stackrel{7}{i}$ & : & $\stackrel{\infty}{\stackrel{\infty}{\sim}}$ \\
\hline 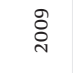 & ठั & 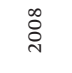 & ڤે & 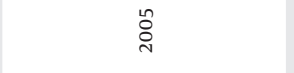 & 㝕 & 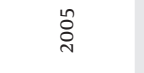 & ఫें & 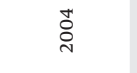 & 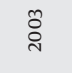 & ర్ర & ठั \\
\hline 芌 & 客 察 & 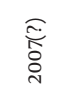 & 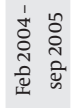 & 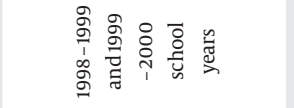 & 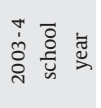 & 总总 & 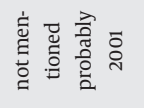 & 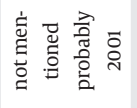 & 芫 & 管 & 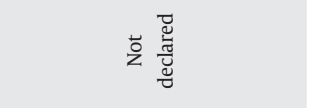 \\
\hline 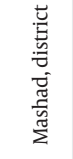 & 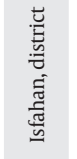 & 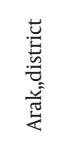 & 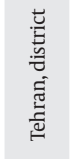 & 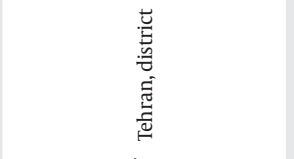 & 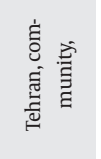 & 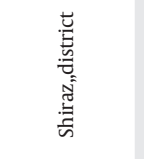 & 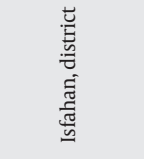 & 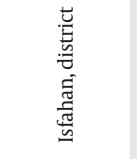 & 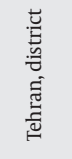 & 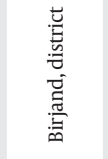 & 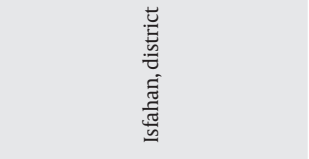 \\
\hline & 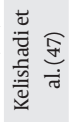 & 㲵 & 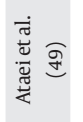 & 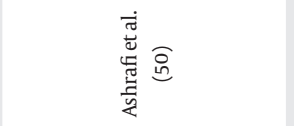 & 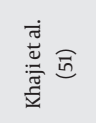 & 胥 & 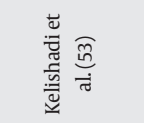 & 离 & 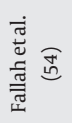 & 蒂焉 & 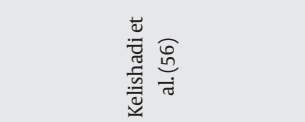 \\
\hline \pm & $ص$ & 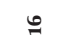 & $=$ & $\Phi$ & 2 & i & $\bar{\pi}$ & $\bar{\pi}$ & $\pi$ & $\approx$ & $\stackrel{d}{\pi}$ \\
\hline
\end{tabular}




\begin{tabular}{|c|c|c|c|c|c|c|c|}
\hline$\Sigma$ & $\S$ & $\frac{\pi}{z}$ & $\bar{z}$ & 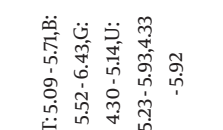 & 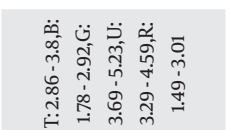 & 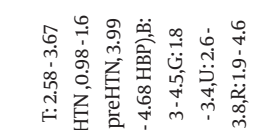 & 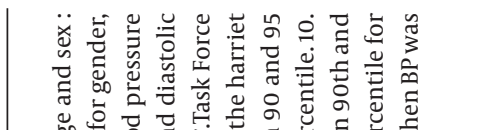 \\
\hline 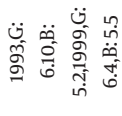 & $\hat{z}$ & $\xi$ & 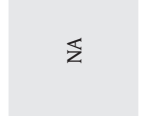 & 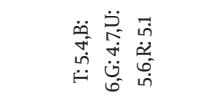 & 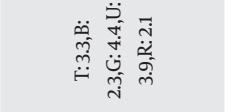 & 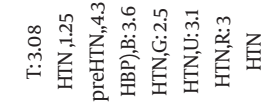 & 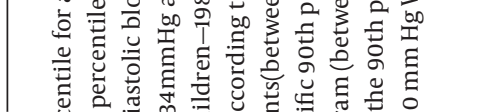 \\
\hline 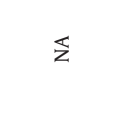 & $\S$ & $\tilde{z}$ & $\bar{z}$ & 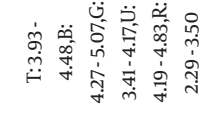 & 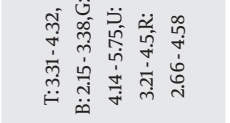 & 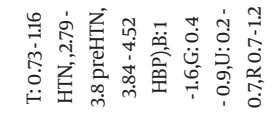 & 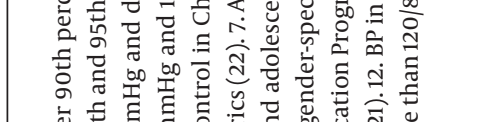 \\
\hline 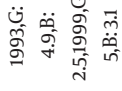 & $\Sigma$ & & $\bar{z}$ & & 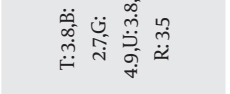 & 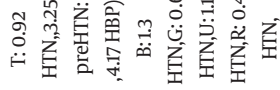 & 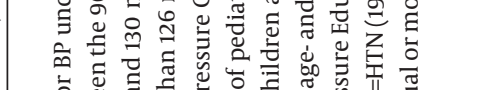 \\
\hline$\Sigma$ & 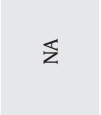 & 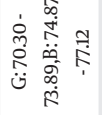 & $\frac{\pi}{z}$ & 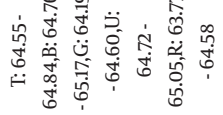 & 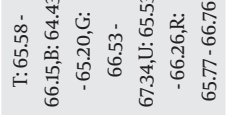 & 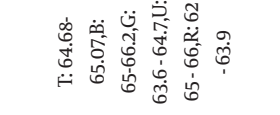 & 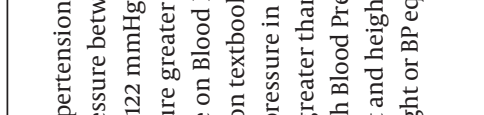 \\
\hline$\frac{\pi}{z}$ & 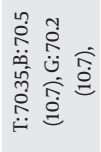 & 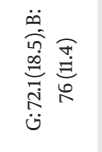 & 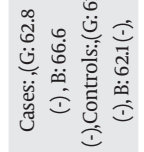 & 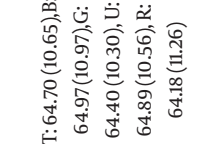 & 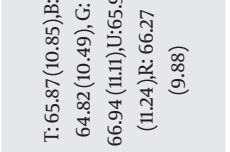 & 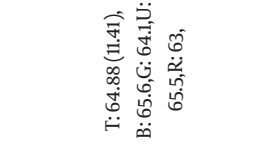 & 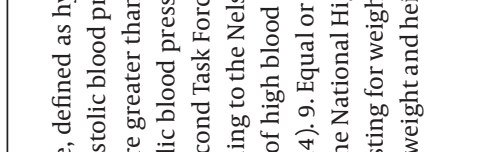 \\
\hline$\Sigma$ & $\bar{z}$ & 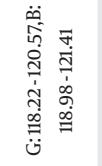 & $\Sigma$ & 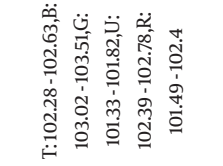 & 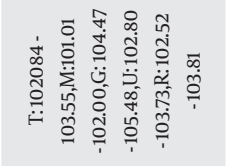 & 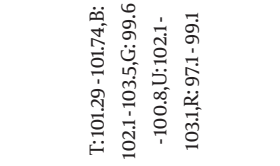 & 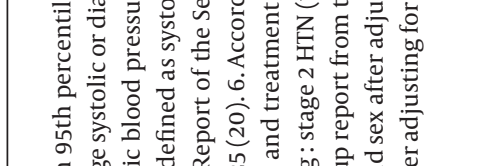 \\
\hline $\bar{z}$ & 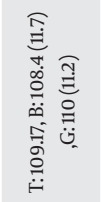 & 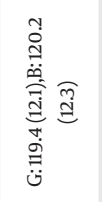 & 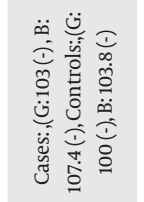 & 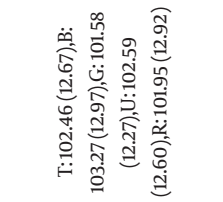 & 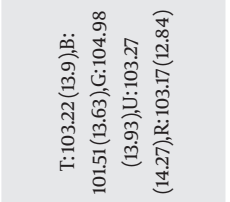 & 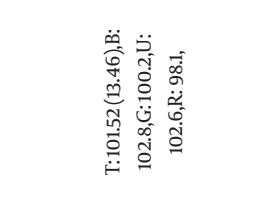 & 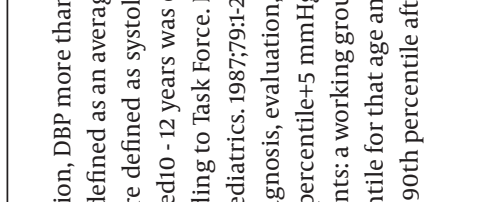 \\
\hline 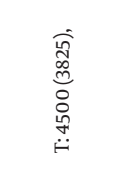 & 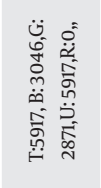 & 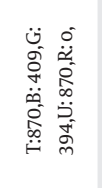 & 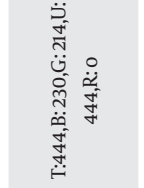 & 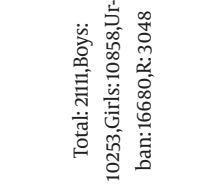 & 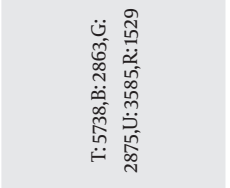 & 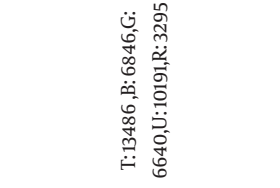 & 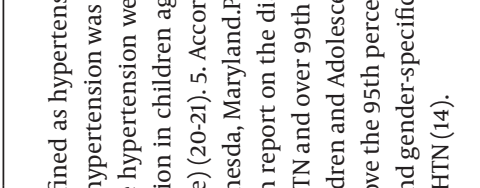 \\
\hline N & $\sim$ & 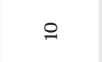 & $\S$ & $=$ & $\simeq$ & 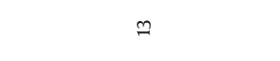 & \\
\hline 营 & 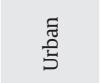 & $\begin{array}{l}\text { 总 } \\
\text { 产 }\end{array}$ & 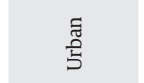 & 暠 & 营 & 营 & \\
\hline 言 & 营 & 营 & 宫 & 咅 & 古 & 咅 & $\underbrace{0}_{3}$ \\
\hline$\Sigma$ & $\Sigma$ & $\bar{z}$ & $\bar{z}$ & $\begin{array}{l}\widehat{\tilde{m}} \\
\stackrel{\pi}{\pi}\end{array}$ & 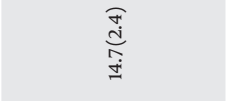 & 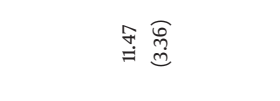 & 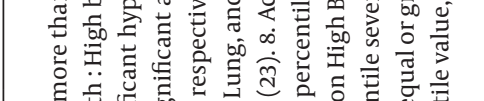 \\
\hline$\stackrel{\infty}{\vec{i}}$ & $\stackrel{?}{\sim}$ & $\stackrel{\square}{ \pm}$ & $\stackrel{\sim}{\check{1}}$ & $\stackrel{\infty}{b}$ & $\stackrel{\infty}{\dot{\omega}}$ & $\stackrel{9}{0}$ & 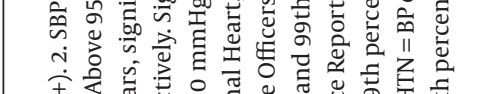 \\
\hline $\overrightarrow{\mathrm{o}}$ & ¿্సి & $\stackrel{\infty}{g}$ & $\stackrel{\infty}{\circ}$ & : & : & ì & 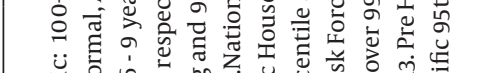 \\
\hline 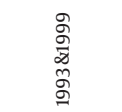 & 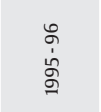 & 宮 & 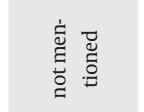 & 雚 薃 & ذ્ّે & 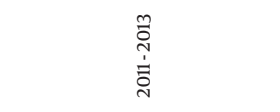 & 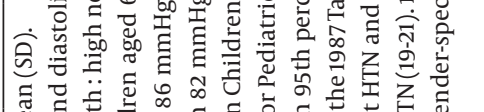 \\
\hline 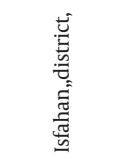 & 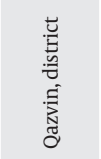 & 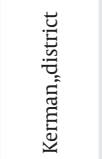 & 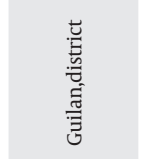 & 胥 & 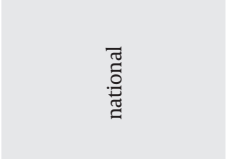 & 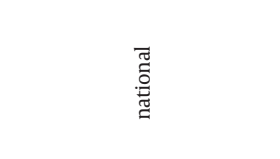 & 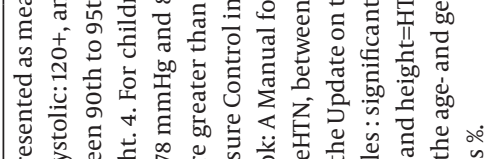 \\
\hline 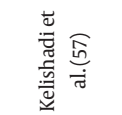 & 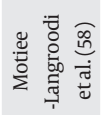 & 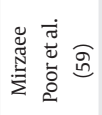 & 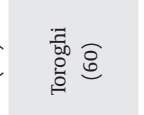 & 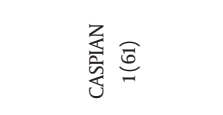 & 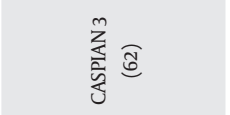 & 妾 & 㐫 \\
\hline in & $\stackrel{\sim}{\circ}$ & กิ & $\stackrel{\sim}{\infty}$ & శి & ㅇ & $\bar{m}$ & \\
\hline
\end{tabular}




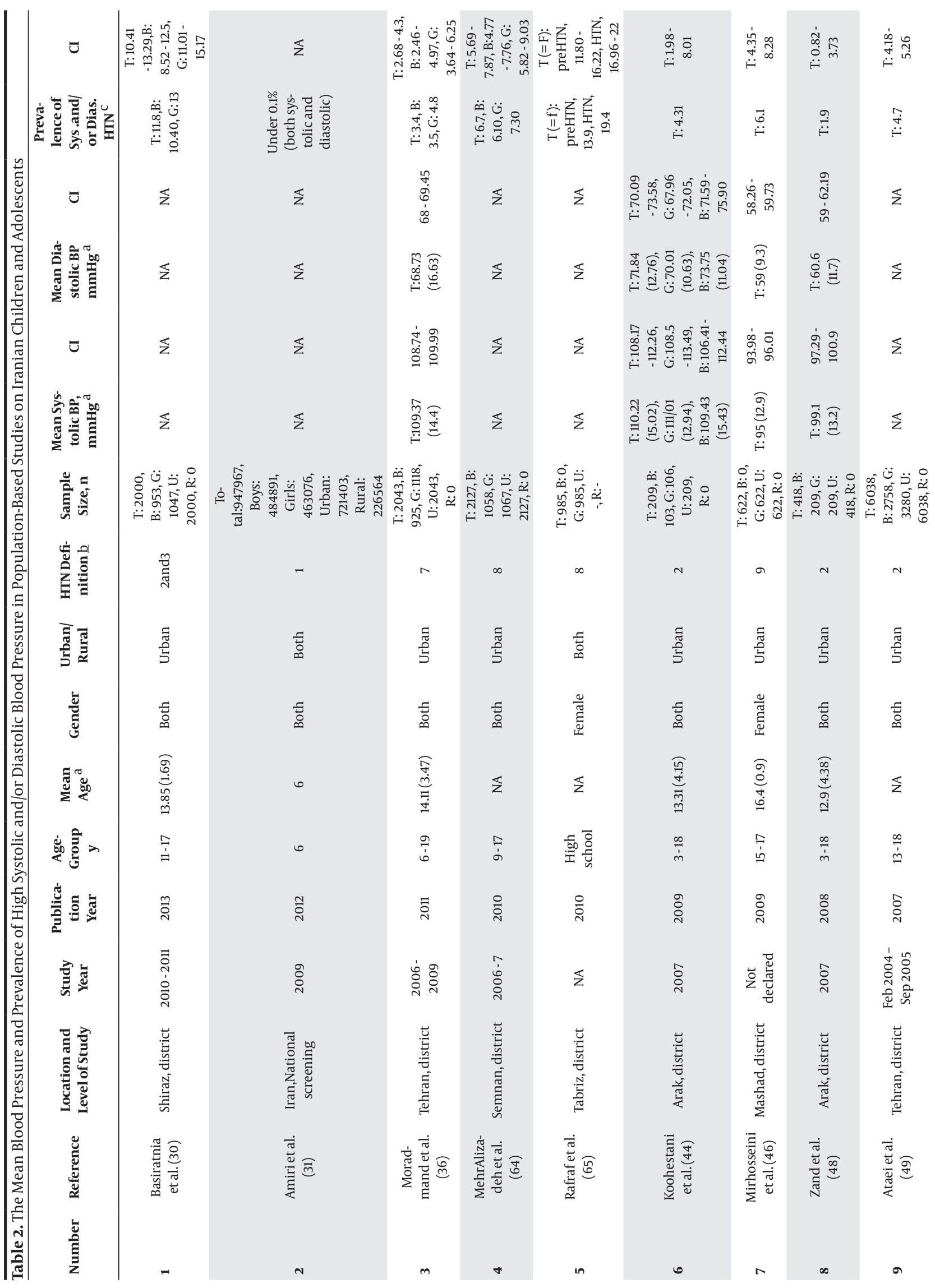




\begin{tabular}{|c|c|c|c|c|c|c|}
\hline 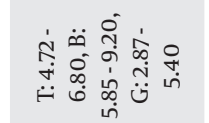 & 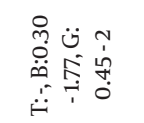 & 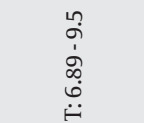 & 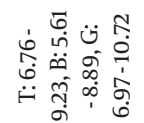 & 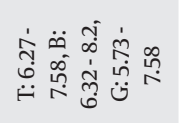 & 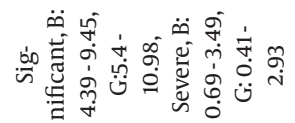 & 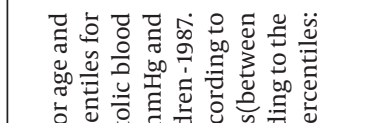 \\
\hline 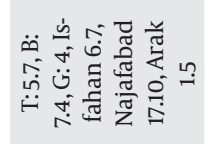 & 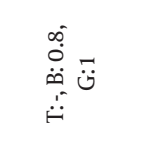 & $\begin{array}{l}\vec{\infty} \\
\stackrel{\leftrightarrow}{H}\end{array}$ & 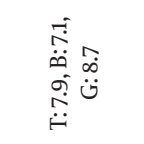 & 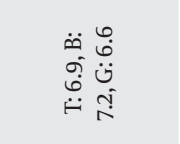 & 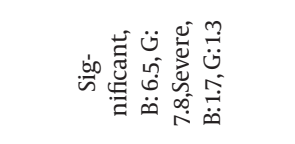 & 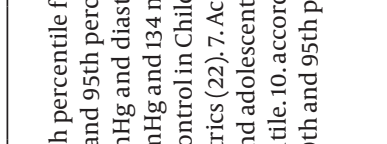 \\
\hline$\S$ & $\S$ & $\S$ & 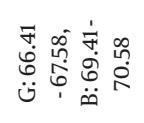 & $\S$ & 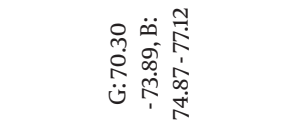 & 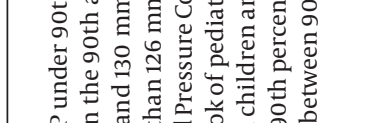 \\
\hline$\overleftarrow{z}$ & 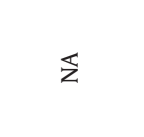 & $\bar{z}$ & 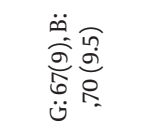 & 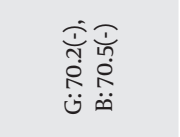 & 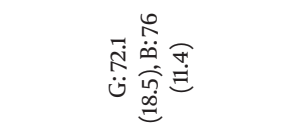 & 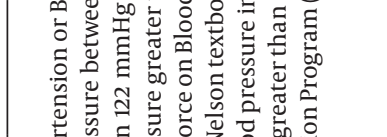 \\
\hline $\mathbb{z}$ & $\Sigma$ & $\S$ & 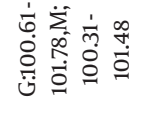 & $\mathbb{z}$ & 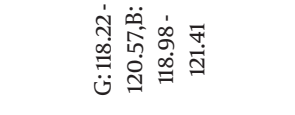 & 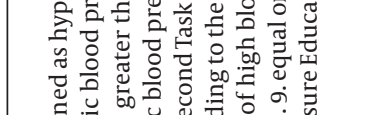 \\
\hline$\Sigma$ & $\frac{\pi}{z}$ & $\Sigma$ & 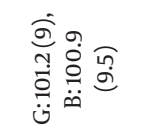 & 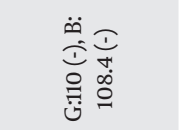 & 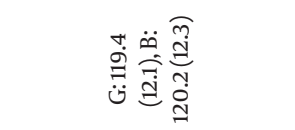 & 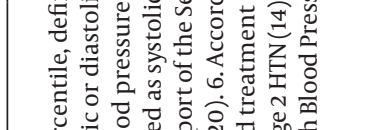 \\
\hline 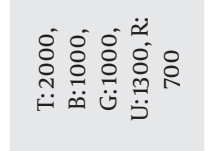 & 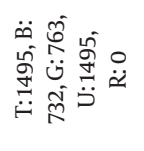 & 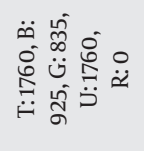 & 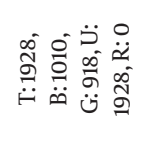 & 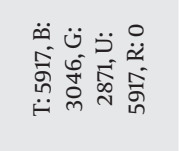 & 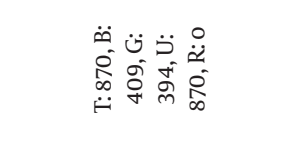 & 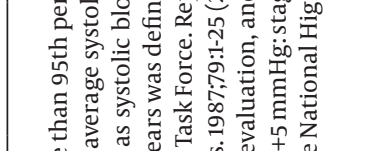 \\
\hline N & 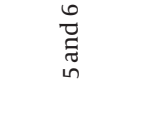 & a & N & N & 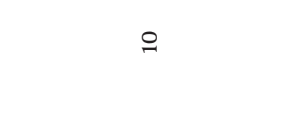 & 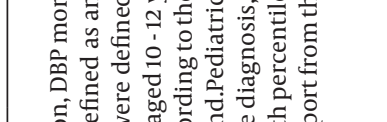 \\
\hline 䒿 & 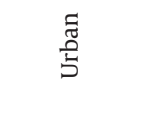 & 营 & $\begin{array}{l}\text { 营 } \\
\text { 点 }\end{array}$ & $\begin{array}{l}\text { 节 } \\
\text { है }\end{array}$ & 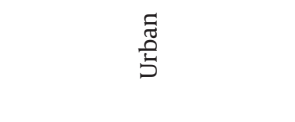 & 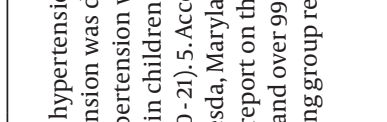 \\
\hline 咅 & 言 & है & 言 & 言 & $\begin{array}{l}\tilde{F} \\
\stackrel{0}{\circ}\end{array}$ & 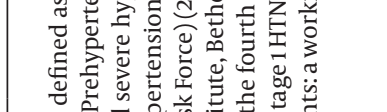 \\
\hline 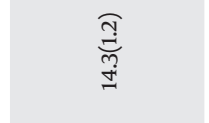 & $\bar{z}$ & $\Sigma$ & $\mathbb{z}$ & $\Sigma$ & $\Sigma$ & 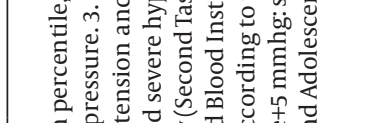 \\
\hline$\stackrel{\infty}{=}$ & $\stackrel{亏}{=}$ & $\stackrel{F}{r}$ & $\stackrel{F}{\sim}$ & $\stackrel{7}{\check{r}}$ & $\stackrel{I}{ \pm}$ & 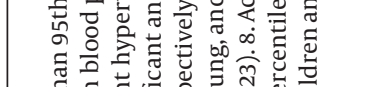 \\
\hline ڤ̊ & ठั & ठั & ठั & 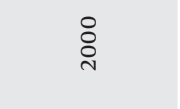 & ळ̊ & 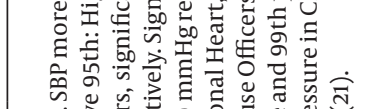 \\
\hline ¿্ণ & פ & 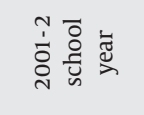 & 号策 & $\begin{array}{l}\text { के } \\
\text { ڤे } \\
\text { ळे }\end{array}$ & $\begin{array}{l}\text { ڤे } \\
\text { ळे }\end{array}$ & 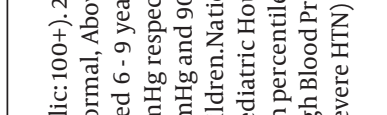 \\
\hline 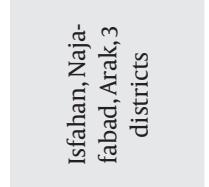 & 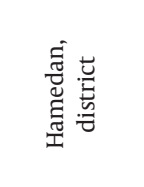 & 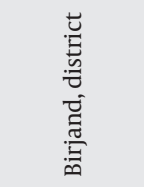 & 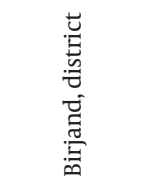 & 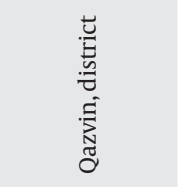 & 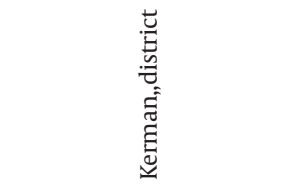 & 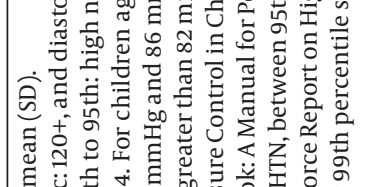 \\
\hline 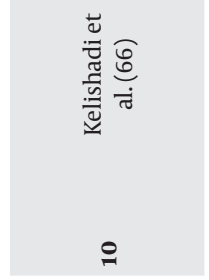 & 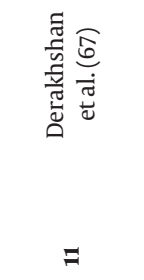 & 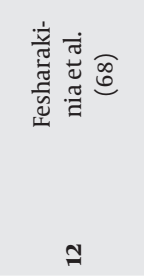 & 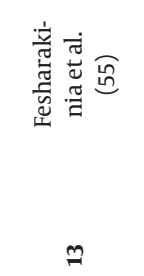 & 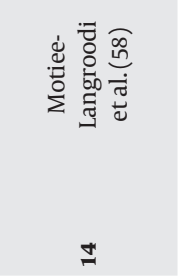 & 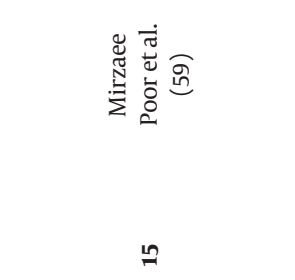 & 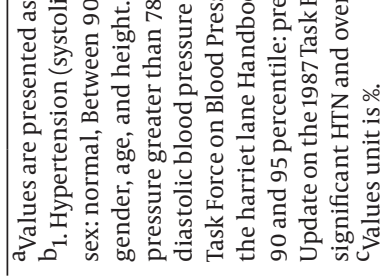 \\
\hline
\end{tabular}




\section{Conclusions}

To the best of our knowledge, this study is the first systematic review on studies related to BP in the pediatric population, not only in Iran, but also, in the MENA region. As expected, we found that the prevalence of elevated BP was not negligible in healthy children and adolescents in Iran, and varied in different regions. It should be considered that in addition to detecting prehypertension and hypertension at early stages, BP screening programs would help in monitoring the mean SBP and DBP over time.

Though a decrease from $41 \%$ in 1990 to $25 \%$ in 2010 has occurred in the contribution of pediatric mortality and morbidity to global DALYs (3), considering that many adult NCDs actually start from earlier stages of life, the importance of this side of pediatric health and its significant sequels in the adult life should not be neglected. Even because of the high susceptibility of fetal life to environmental and nutritional disorders, preventive measures should focus on this stage of life (70). The childhood obesity epidemic has resulted in left ventricular hypertrophy and evidence for premature development of atherosclerosis, therefore it makes the issue of tracking $\mathrm{BP}$ and screening for elevated $\mathrm{BP}$ of special concern in the pediatric age group (71).

Children and adolescents with pre-hypertension and hypertension tend to maintain this situation over time (1, 72-76). Many risky behaviors as smoking, alcohol use, physical inactivity, and unhealthy dietary habits are developed during adolescence period. Changing such lifestyle habits would be difficult after establishment, and would have lifelong consequences including NCDs and their risk factors as high $\mathrm{BP}(77)$. A longitudinal study with 17 years of follow up of a pediatric population showed a 6.88 fold increase in the prevalence of hypertension in those who had high BP at baseline (78).

National studies in American children and adolescents found that that the mean BP levels (79), as well as the prevalence of high BP (80) are gradually rising over time. The situation in Iran seems similar. The mean age of Iranian population is estimated to be increasing by 6.5 years in the two coming decades. Presuming the persistent prevalence of risk and protective factors and only because of that increase in the age, the country's YLL from CVDs will be duplicated in 2025 compared to 2005, without difference in terms of gender (81). A Study in 2003 showed that in Iran 58 percent of DALYs resulted from NCDs and the country health burden is changing from communicable diseases to NCDs and road accidents (82). WHO has reported an agestandardized mortality rate of 420.8 (males) and 348.0 (females) per 100000 for CVD and diabetes in Iran for 2008, and estimated a prevalence rate of around 33.7\% for hypertension (7). A national survey (SuRFNCD) in 2007 showed a remarkable prevalence of NCDs and their risk factors among Iranians aged 15 - 65 (83). Another national study showed that high SBP is responsible for most deaths in all regions of Iran. It is expected that by optimizing SBP, the years of life expectancy would increase by 3.2 (2.6, 3.9 95\% uncertainty interval) years in men and by 4.1 (3.2, 4.9 UI) years in women, therefore it is concluded that prevention and control of high BP should be considered as a health priority for Iran (84). Many studies documented high rates of cardio-metabolic predisposing factors in Iranian pediatric population (25-29, 85-90). The CASPIAN-I study (25, 26), showed that the percentiles of SBP and DBP of Iranian children and adolescents were in close agreement with reference values (14). As mentioned earlier, the study has continued to the 4th survey till now and different rates of elevated BP are reported. Overall, these surveys showed that in recent years, the prevalence of elevated BP has increased slightly. The variation between surveys could be attributed to differences between definitions of elevated BP (the first phase was conducted before the introduction of pediatric pre-HTN) and differences in study populations, i.e. different proportion of age and gender subgroups, different provinces, different total populations and changes in the prevalence of associated conditions as obesity, unhealthy dietary habits, inactivity, air and noise pollution, urbanization, etc. $(69,91)$.

Given the association of BP level with excess weight as well as with environmental factors as air pollution, noise pollution, and passive smoking (91), and the high sodium intake of Iranian children (33), it is proposed that in the near future, the mean BP and the prevalence of high BP will be escalating in Iranian children and adolescents.

The high prevalence of NCD risk factors in various age groups of Iranians, underscores the necessity of conducting comprehensive research and preventive programs to achieve a multidisciplinary intervention plan involving the whole health system of Iran (92). Both in the 53rd session of Eastern Mediterranean Region (EMRO) (93) and September 2011 session of the United Nations (UN) in New York, all members committed to develop national strategies as well as preventive and controlling action plans, to involve all stakeholders and to organize financial resources targeting NCDs. It seems that still no effective public and community interventions have been done in this regard. The GBD study 2010 revealed that some parts of underachievement of the WHO 4th millennium goal is because of the lack of relevant information from the pediatric age group (94). Presenting the information regarding pediatric studies to policymakers in a systematic and concluding manner would be of help for planning action-oriented programs. Moreover, there is increasing need to know where and how the complementary research and action plans must be implemented.

The current study summarized the information on the mean BP and the prevalence of high BP in the Iranian pediatric population. The wide heterogeneity of the retrieved papers and their point estimates led the researchers not to consider a meta-analysis. It is planned to draw the trend of BP changes across these years. As mentioned 
before, we will use advanced statistical modeling for missed data handling and estimations. After that, YLDs, YLLs, DALYs and burden of the HBP in pediatric population of Iran will be estimated.

The importance of BP tracking and the pediatric hypertension per se and as a CVD risk factor becomes more obvious, making it important for including BP measurement in screening programs and conducting further comprehensive and cumulative studies on its time trends, primary prevention and early diagnosis.

Health policies on prevention and early control of high $\mathrm{BP}$ can be effective in reducing the prevalence and the adverse consequences of high BP in adulthood. Therefore, the findings of the current systematic review and succeeding works on BP of Iranian children and adolescents would be useful for future health policies and research activities aimed to reduce the burden of high BP at individual and public health levels. Follow up surveillance programs and comparison of BP trends of children and adolescents over time are recommended.

\section{Footnote}

Authors' Contribution:Zahra Fallah: acquisition of data, data interpretation, drafting of the manuscript, critical revision of the manuscript and approval of the article. Shirin Djalalinia: critical revision of the manuscript and approval of the article. Mostafa Qorbani: include contributions to concept/design, data analysis/interpretation, critical revision of the manuscript and approval of the article. Farshad Farzadfar: include contributions to concept/design, data analysis/interpretation, critical revision of the manuscript and approval of the article. Roya Kelishadi: include contributions to concept/design, data interpretation, drafting of the manuscript, critical revision of the manuscript and approval of the article.

\section{Appendices}

Please visit article's online version for appendices.

\section{References}

1. Murray CJ, Ezzati M, Flaxman AD, Lim S, Lozano R, Michaud C, et al. GBD 2010: design, definitions, and metrics. Lancet. 2012;380(9859):2063-6. doi: 10.1016/S0140-6736(12)61899-6. [PubMed: 23245602]

2. Lim SS, Vos T, Flaxman AD, Danaei G, Shibuya K, Adair-Rohani H, et al. A comparative risk assessment of burden of disease and injury attributable to 67 risk factors and risk factor clusters in 21 regions, 1990-2010: a systematic analysis for the Global Burden of Disease Study 2010. Lancet. 2012;380(9859):2224-60. doi:10.1016| S0140-6736(12)61766-8. [PubMed:23245609]

3. Wang H, Dwyer-Lindgren L, Lofgren KT, Rajaratnam JK, Marcus JR, Levin-Rector A, et al. Age-specific and sex-specific mortality in 187 countries, 1970-2010: a systematic analysis for the Global Burden of Disease Study 2010. Lancet. 2012;380(9859):2071-94. doi: 10.1016/S0140-6736(12)61719-X. [PubMed: 23245603]

4. Salomon JA, Wang H, Freeman MK, Vos T, Flaxman AD, Lopez AD, et al. Healthy life expectancy for 187 countries, 1990-2010: a sys- tematic analysis for the Global Burden Disease Study 2010. Lancet. 2012;380(9859):2144-62. doi: 10.1016/S0140-6736(12)61690-0. [PubMed: 23245606]

5. Vos T, Flaxman AD, Naghavi M, Lozano R, Michaud C, Ezzati M, et al. Years lived with disability (YLDs) for 1160 sequelae of 289 diseases and injuries 1990-2010: a systematic analysis for the Global Burden of Disease Study 2010. Lancet. 2012;380(9859):2163-96. doi:10.1016/S0140-6736(12)61729-2. [PubMed:23245607]

6. Lozano R, Naghavi M, Foreman K, Lim S, Shibuya K, Aboyans V, et al. Global and regional mortality from 235 causes of death for 20 age groups in 1990 and 2010: a systematic analysis for the Global Burden of Disease Study 2010. Lancet. 2012;380(9859):2095-128. doi:10.1016/S0140-6736(12)61728-0. [PubMed: 23245604]

7. Lawes CM, Vander Hoorn S, Rodgers A, International Society of H. Global burden of blood-pressure-related disease, 2001. Lancet. 2008;371(9623):1513-8. doi: 10.1016/S0140-6736(08)60655-8. [PubMed:18456100]

8. Mathers CD, Ezzati M, Lopez AD. Measuring the burden of neglected tropical diseases: the global burden of disease framework. PLoS Negl Trop Dis. 2007;1(2):e4496 doi: 10.1371/journal. pntd.0000114. [PubMed: 18060077]

9. Global Health Risks. WHO Press. Mortality and burden of disease attributable to selected major risks. 2009

10. World Health Organization. Noncommunicable diseases country profiles 2011, WHO global report, 2012. 2013 Available from: www. who.int.

11. Balagopal PB, de Ferranti SD, Cook S, Daniels SR, Gidding SS, Hayman LL, et al. Nontraditional risk factors and biomarkers for cardiovascular disease: mechanistic, research, and clinical considerations for youth: a scientific statement from the American Heart Association. Circulation. 2011;123(23):2749-69. doi: 10.1161/ CIR.ob013e31821c7c64. [PubMed: 21555711]

12. Sun SS, Grave GD, Siervogel RM, Pickoff AA, Arslanian SS, Daniels SR. Systolic blood pressure in childhood predicts hypertension and metabolic syndrome later in life. Pediatrics. 2007;119(2):23746. doi: 10.1542/peds.2006-2543. [PubMed: 17272612]

13. Li S, Chen W, Srinivasan SR, Berenson GS. Childhood blood pressure as a predictor of arterial stiffness in young adults: the bogalusa heart study. Hypertension. 2004;43(3):541-6. doi: 10.1161/01. HYP.0000115922.98155.23. [PubMed:14744922]

14. National High Blood Pressure Education Program Working Group on High Blood Pressure in C, Adolescents. The fourth report on the diagnosis, evaluation, and treatment of high blood pressure in children and adolescents. Pediatrics. 2004;114(2 Suppl 4th Report):555-76. [PubMed:15286277]

15. Larijani F, Parsaeian M, Kalantar Motamedi SM, Khosravi A, Farzadfar F, Larijani B. National trends in mortality attributable to metabolic risk factors in Iran. Lancet. 2013;381:S79. doi: 10.1016| s0140-6736(13)61333-1.

16. Parsaeian M, Farzadfar F, Zeraati H, Mahmoudi M, Rahimighazikalayeh G, Navidi I, et al. Application of spatio-temporal model to estimate burden of diseases, injuries and risk factors in Iran 1990 - 2013. Arch Iran Med. 2014;17(1):28-33. [PubMed:24444062]

17. Kasaeian A, Eshraghian MR, Rahimi Foroushani A, Niakan Kalhori SR, Mohammad K, Farzadfar F. Bayesian autoregressive multilevel modeling of burden of diseases, injuries and risk factors in Iran 1990 - 2013. Arch Iran Med. 2014;17(1):22-7. [PubMed: 24444061]

18. Farzadfar F, Delavari A, Malekzadeh R, Mesdaghinia A, Jamshidi HR, Sayyari A, et al. NASBOD 2013: design, definitions, and metrics. Arch Iran Med. 2014;17(1):7-15. [PubMed: 24444059]

19. Kelishadi R, Hovsepian S, Qorbani M, Jamshidi F, Fallah Z, Djalalinia $S$, et al. National and sub-national prevalence, trend, and burden of cardiometabolic risk factors in Iranian children and adolescents, 1990 - 2013. Arch Iran Med. 2014;17(1):71-80. [PubMed: 24444066]

20. Report of the Second Task Force on Blood Pressure Control in Children-1987. Task Force on Blood Pressure Control in Children. National Heart, Lung, and Blood Institute, Bethesda, Maryland. Pediatrics. 1987;79(1):1-25. [PubMed: 3797155]

21. Update on the 1987 Task Force Report on High Blood Pressure in Children and Adolescents: a working group report from the Na- 
tional High Blood Pressure Education Program. National High Blood Pressure Education Program Working Group on Hypertension Control in Children and Adolescents. Pediatrics.1996;98(4 Pt 1):649-58. [PubMed: 8885941]

22. Behrman RE, Kliegman RM, Jenson HB. Nelson Textbook of Pediatrics. 16 th ed. Philadelphia: WB Saunders; 2000.

23. Gunn VL, Nechyba CH. The Harriet Lane Handbook: A Manual for Pediatrics House Officers. 16th ed. Philadelphia: Mosby; 2002.

24. WHO. Global School-Based Student Health Survey (GSHS). Available from: http://www.who.int/chp/gshs/en/.

25. Motlagh ME, Kelishadi R, Ardalan G, Gheiratmand R, Majdzadeh $\mathrm{R}$, Heidarzadeh A, et al. Rationale, methods and first results of the Iranian national programme for prevention of chronic diseases from childhood: CASPIAN Study. East Mediterr Health J 2009;15(2):302-14. [PubMed: 19554976]

26. Kelishadi R, Amirkhani A, Ardalan G, Ziaoddini H, Majdzadeh R. An overview of a national surveillance program in iran for prevention of chronic non-communicable diseases from childhood: CASPIAN study. Iran J Pub Health. 2009;38(Suppl. 1):102-6.

27. Amirkhani A, Motlagh ME, Sedaghat M, Namazi R, Ardalan G, Haghdust A, et al. Health status of Iranian students according to the health-risk behaviors survey 2006-2007. Iran: Publications of the Ministry of Health and Medical Education,; 2009.

28. Kelishadi R, Heshmat R, Motlagh ME, Majdzadeh R, Keramatian K, Qorbani M, et al. Methodology and Early Findings of the Third Survey of CASPIAN Study: A National School-based Surveillance of Students' High Risk Behaviors. Int J Prev Med. 2012;3(6):394-401. [PubMed: 22783465]

29. Kelishadi R, Ardalan G, Oorbani M, Ataie-Jafari A, Bahreynian M, Taslimi M, et al. Methodology and Early Findings of the Fourth Survey of Childhood and Adolescence Surveillance and Prevention of Adult Non-Communicable Disease in Iran: The CASPIANIV Study. Int J Prev Med. 2013;4(12):1451-60. [PubMed:24498502]

30. Basiratnia M, Derakhshan D, Ajdari S, Saki F. Prevalence of childhood obesity and hypertension in south of Iran. Iran J Kidney Dis 2013;7(4):282-9. [PubMed: 23880805]

31. Amiri M, Kelishadi R, Motlagh ME, Taslimi M, Taheri M, Ardalan G et al. Prevalence study of clinical disorders in 6-year-old children across Iranian provinces: Findings of Iranian national health assessment survey. J Res Med Sci. 2012;17(7):596-601. [PubMed: 23798916]

32. Seyedzadeh A, Hashemi F, Soleimani A. Relationship between Blood Pressure and Passive Smoking in Elementary School Children. Iran JPediatr. 2012;22(3):351-6. [PubMed: 23400119]

33. Kelishadi R, Gheisari A, Zare N, Farajian S, Shariatinejad K. Salt intake and the association with blood pressure in young Iranian children: first report from the middle East and north Africa. Int J Prev Med. 2013;4(4):475-83. [PubMed: 23671781]

34. Shajari H, Shajari A, Sepahi MA, Mehrparvar AH, Roghani R, Nakhaei MH. Relationship between arterial blood pressure and body mass index of school age children of southern region of Iran. Acta Med Iran. 2011;49(11):737-41. [PubMed: 22131244]

35. Mohkam M, Karimi A, Eslami N, Khatami A, Fallah F, Maham S, et al. Blood pressure screening in school-aged children in Tehran. Iran J Kidney Dis. 2011;5(4):229-33. [PubMed: 21725178]

36. Moradmand S, Ganji M, Meisami AP. Frequency of high blood pressure and its relationship with birth and current weight in Tehran city school students [in Persian]. Peyavard-e-Salamat J. 2011-2012;5(4):16-23.

37. Mehrkash M, Mohammadian S, Qorbani M. Prevalence of metabolic syndrome among adolescents aged 15 to 17 years in Gorgan, Northern Iran (2009) [in Persian]. Gorgan Uni Med Sci J. 2010;13(2):93-9.

38. Mehrkash M, Kelishadi R, Mohammadian S, Mousavinasab F, Qorbani M, Hashemi ME, et al. Obesity and metabolic syndrome among a representative sample of Iranian adolescents. Southeast Asian J Trop Med Public Health. 2012;43(3):756-63. [PubMed: 23077856]

39. Hosseini-Esfahani F, Mousavi Nasl Khameneh A, Mirmiran P, Ghanbarian A, Azizi F. Trends in risk factors for cardiovascular disease among Iranian adolescents: the Tehran Lipid and Glucose Study, 1999-2008. J Epidemiol. 2011;21(5):319-28. doi: 10.2188/
jea.JE20100162. [PubMed: 21804294]

40. Ghanbarian A, Salehi P, Rezaei-Ghaleh N. Pattern of blood pressure in urban community of Tehranian adolescents:TLGS [in Persian]. Hakim Res J. 2003;6(3):21-8.

41. Mousavin-asl-Khameneh A, Hosseini-Esfahani F, Safarkhani M. Overweight and hypertension prevalence trend from three surveys of TLGS (2000-2009) [in Persian]. Iran J Endocrinol Metab. 2011;12(5):483-92.

42. Salem Z. Prevalence of hypertension and its relationship with an thropometric measures in adolescent girls of Rafsanjan city in 2007-8 [in persian]. Rafsanjan Med Uni J. 2010;8(4):273-86.

43. Salem Z, Vazirinejad R. Prevalence of some components of metabolic syndrome in 7-11 years old children of Rafsanjan city, 2008-9 [in Persian]. Iran Nutr Sci Food Industries J. 2010;5(2):63-71.

44. Koohestani HR, Zand S, Baghchaghi N, Rezaei K. Atherosclerosis risk factors frequency in offsprings of persons with premature cardiac attack [in Persian]. Zanjan Medl Uni Sci-Res J. 2010;17(69):35-44

45. Mahyar A, Ebrahemi M, Shahsavari A, Rahmani Y. Blood pressure of primary-school children of Eghbalieh city, Islamic Republic of Iran. East Mediterr Health J. 2009;15(6):1449-54. [PubMed: 20218137]

46. Mirhosseini NZ, Yusoff NA, Shahar S, Parizadeh SM, Mobarhen MG, Shakery MT. Prevalence of the metabolic syndrome and its influencing factors among adolescent girls in Mashhad, Iran Asia Pac J Clin Nutr. 2009;18(1):131-6. [PubMed:19329406]

47. Kelishadi R, Cook SR, Adibi A, Faghihimani Z, Ghatrehsamani S, Beihaghi A, et al. Association of the components of the metabolic syndrome with non-alcoholic fatty liver disease among normalweight, overweight and obese children and adolescents. Diabetol Metab Syndr. 2009;1:29. doi: 10.1186/1758-5996-1-29. [PubMed 20028551]

48. Zand S, Koohestani HR, Zand S. Comparison of lipid profile and blood pressure of 3-18 year-old children and adolescents with control group [in Persian]. Pediatr Dis J. 2009;18((Supl 1)):59-64.

49. Ataei N, Aghamohammadi A, Hoseini M. Hypertension prevalence in 13-18 year old students in Iran [in Persian]. Iran Pediatr Dis J. 2008;17(Supl 2):237-42.

50. Ashrafi MR, Abdollahi M,Ahranjani BM, Shabanian R. Blood pressure distribution among healthy schoolchildren aged 6-13 years in Tehran. East Mediterr Health J. 2005;11(5-6):968-76. [PubMed: 16761667]

51. Khaji A, Khodaei S, Karbakhsh M. hypertension and obesity in Tehrani adolescents [in Persian]. Iran Pediatric Dis J. 2007;16(1):4550.

52. Ayatollahi SM, Zare M. Determinants of hypertension in elementary school children of Shiraz (2004) [in Persian]. Behbood Quart Sci-Res J Kermanshah Medl Uni. 2008;11(84):376-85.

53. Kelishadi R, Hashemipour M, Bashardoost N. Blood pressure in children of hypertensive and normotensive parents. Indian Pedi atr. 2004;41(1):73-7. [PubMed: 14767089]

54. Fallah A, Gachkar L, Faraji S. Evaluation of blood pressure in 7-1 year-old school children of Tehran city in the year 2003-4 [in persian]. Zanjan Uni Medical Sci Health Serv Sci J. 2004;42:43-8.

55. Fesharakinia A, Taheri F, Saadatjoo SA. Relationship of blood pressure with BMI in 7-11 year-old children of Birjand district [in persian]. Birjand Med Uni J. 2002;8(1)

56. Kelishadi R, Zadegan NS, Naderi GA, Asgary S, Bashardoust N. Atherosclerosis risk factors in children and adolescents with or without family history of premature coronary artery disease. Med Sci Monit. 2002;8(6):CR425-9. [PubMed:12070433]

57. Kelishadi R, Hashemipour M, Sarraf-Zadegan N, Amiri M. Trend of Atherosclerosis Risk Factors in Children of Isfahan. Asian CardiovascThoracAnn.2001;9(1):36-40.doi:10.1177/021849230100900110.

58. Motiei-Langroodi SH, Ghoreishi SG, Asgari FA, Kavian K. Blood pressure and pulse rate of 7 to 11 year-old children of Qazvin district schools [in persian]. Qazvin Uni Med Sci Health Serv J. 2001;Spring:62-5.

59. Mirzaeipoor F, Azimzadeh BS, Moshiri M, Dehghani S. Evaluation of blood pressure and its relationship with BMI in Kerman city high school students (Autumn 1997) [in Persian]. Feiz Quart SciRes J. 1998;5:77-89. 
Fallah Z et al.

60. Toroghi M. Comparison between blood pressure of 6-12 year-old children of parents with essential hypertension and children of parents with normal blood pressure [in Persian]. Zanjan Uni Med Sci Health Serv J. 1998;24:31-6.

61. Kelishadi R, Ardalan G, Gheiratmand R, Majdzadeh R, Delavari A, Heshmat R, et al. Blood pressure and its influencing factors in a national representative sample of Iranian children and adolescents: the CASPIAN Study. Eur J Cardiovasc Prev Rehabil. 2006;13(6):956-63. doi: 10.1097/01.hjr.0000219109.17791.b6. [PubMed:17143128]

62. Khashayar P, Heshmat R, Qorbani M, Motlagh ME, Aminaee T, Ardalan G, et al. Metabolic Syndrome and Cardiovascular Risk Factors in a National Sample of Adolescent Population in the Middle East and North Africa: The CASPIAN III Study. Int J Endocrinol. 2013;2013:1-8. doi: 10.1155/2013/702095.

63. Fallah Z, Qorbani M, Motlagh ME, Heshmat R, Ardalan G, Kelishadi R. Prevalence of Prehypertension and Hypertension in a Nationally Representative Sample of Iranian Children and Adolescents: The CASPIAN-IV Study. Int JPrev Med. 2014;5(Suppl1):S57-64. [PubMed: 24791193]

64. Mehralizadeh S, Ghorbani R, Sharafi S. Prevalence of high blood pressure in 9-17 year-old students of Semnan [in Persian]. Semnan Med Uni J. 2010;12(1):1-8.

65. Rafraf M, Gargari BP, Safaiyan A. Prevalence of prehypertension and hypertension among adolescent high school girls in Tabriz, Iran. Food Nutr Bull. 2010;31(3):461-5. [PubMed: 20973466]

66. Kelishadi R, Sadri G, Tavasoli AA, Kahbazi M, Roohafza HR, Sadeghi M, et al. Cumulative prevalence of risk factors for atherosclerotic cardiovascular diseases in Iranian adolescents: IHHPHHPC. J Pediatr (Rio J). 2005;81(6):447-53. doi: 10.2223/JPED.1418. [PubMed: 16385361]

67. Derakhshan M. Evaluation of blood pressure and its relationship with height and weight in 7-11 year-old students of Hamedan city [in persian]. Res Med Sci J. 2003;26(2):92-6.

68. Fesharakinia A, Taheri F, Saadatjoo SA. Blood pressure normogram of elementary school students of Birjand [in Persian]. Birjand Med Uni J. 2001;7(1)

69. Falkner B. Hypertension in children and adolescents: epidemiology and natural history. Pediatr Nephrol. 2010;25(7):1219-24. doi: 10.1007/s 00467-009-1200-3. [PubMed:19421783]

70. Murray CJ, Vos T, Lozano R, Naghavi M, Flaxman AD, Michaud C, et al. Disability-adjusted life years (DALYs) for 291 diseases and injuries in 21 regions, 1990-2010: a systematic analysis for the Global Burden of Disease Study 2010. Lancet. 2012;380(9859):2197-223. doi:10.1016/S0140-6736(12)61689-4. [PubMed: 23245608]

71. Barouki R, Gluckman PD, Grandjean P, Hanson M, Heindel JJ. Developmental origins of non-communicable disease: implications for research and public health. Environ Health. 2012;11:42. doi:10.1186/1476-069X-11-42. [PubMed: 22715989]

72. Luma GB, Spiotta RT. Hypertension in children and adolescents Am Fam Physician. 2006;73(9):1558-68. [PubMed:16719248]

73. Long AN, Dagogo-Jack S. Comorbidities of diabetes and hypertension: mechanisms and approach to target organ protection. J Clin Hypertens (Greenwich). 2011;13(4):244-51. doi: 10.1111/j.17517176.2011.00434.x. [PubMed: 21466619]

74. Chen X, Wang Y. Tracking of blood pressure from childhood to adulthood: a systematic review and meta-regression analysis. Circulation. 2008;117(25):3171-80. doi: 10.1161/CIRCULATIONAHA.107.730366. [PubMed:18559702]

75. Gidding SS. Measuring children's blood pressure matters. Circulation. 2008;117(25):3163-4. doi: 10.1161/CIRCULATIONAHA.108.787168. [PubMed: 18574056]

76. Bao W, Threefoot SA, Srinivasan SR, Berenson GS. Essential hy pertension predicted by tracking of elevated blood pressure from childhood to adulthood: the Bogalusa Heart Study. Am J Hypertens. 1995;8(7):657-65. doi: 10.1016/0895-7061(95)00116-7. [PubMed: 7546488]

77. Fuentes RM, Notkola IL, Shemeikka S, Tuomilehto J, Nissinen A Tracking of systolic blood pressure during childhood: a 15-year follow-up population-based family study in eastern Finland. J Hy- pertens. 2002;20(2):195-202. [PubMed: 11821703]

78. AstraZeneca Young Health Programme. Non-communicable diseases and adolescents: an opportunity for action. 2011 Available from: http://www.jhsph.edu/bin/g/q/ noncommunicable.pdf.

79. Mu JJ, Liu ZQ, Yang J, Ren J, Liu WM, Xu XL, et al. Association between higher blood pressure level in children and adult blood pressure: 17 years follow-up results [in Chinese]. Zhonghua Xin Xue Guan Bing Za Zhi. 2008;36(3):229-31. [PubMed:19099979]

80. Flynn JT, Falkner BE. The importance of blood pressure screening in children. J Pediatr. 2009;155(2):299-300. doi: 10.1016/j. jpeds.2009.03.037. [PubMed: 19619758]

81. Din-Dzietham R, Liu Y, Bielo MV, Shamsa F. High blood pressure trends in children and adolescents in national surveys, 1963 to 2002. Circulation. 2007;116(13):1488-96. doi: 10.1161/CIRCULATIONAHA.106.683243. [PubMed:17846287]

82. Sadeghi M. Modeling for the impact of age and sex pyramid changes on cardiovascular diseases burden during two coming decades in Iran [in Persian].J Knowl Health. 2010;5:66.

83. Naghavi M, Abolhassani F, Pourmalek F, Lakeh M, Jafari N, Vaseghi $\mathrm{S}$, et al. The burden of disease and injury in Iran 2003. Popul Health Metr. 2009;7:9. doi:10.1186/1478-7954-7-9. [PubMed: 19527516]

84. Esteghamati A, Meysamie A, Khalilzadeh O, Rashidi A, Haghazali M, Asgari F, et al. Third national Surveillance of Risk Factors of Non-Communicable Diseases (SuRFNCD-2007) in Iran: methods and results on prevalence of diabetes, hypertension, obesity, central obesity, and dyslipidemia. BMC Public Health. 2009;9:167. doi:10.1186/1471-2458-9-167. [PubMed:19480675]

85. Farzadfar F, Danaei G, Namdaritabar H, Rajaratnam JK, Marcus JR, Khosravi A, et al. National and subnational mortality effects of metabolic risk factors and smoking in Iran: a comparative risk assessment. Popul Health Metr. 2011;9(1):55. doi: 10.1186/1478-79549-55. [PubMed: 21989074]

86. Esteghamati A, Abbasi M, Alikhani S, Gouya MM, Delavari A Shishehbor MH, et al. Prevalence, awareness, treatment, and risk factors associated with hypertension in the Iranian population: the national survey of risk factors for noncommunicable diseases of Iran. Am J Hypertens. 2008;21(6):620-6. doi: 10.1038| ajh.2008.154. [PubMed:18451810]

87. Motlagh ME, Kazemeini H, Jafari N, Delavar B, Kabir MJ, Godarzi K. Disease burden of 0-14 years old children in Iran [in Persian]. Ofogh-e-Danesh. 2009;15(4):77-85.

88. Poorolajal J, Esmailnasab N, Ahmadzadeh J, Motlagh T. The burden of premature mortality in Hamadan province in 2006 and 2010 using standard expected years of potential life lost: a population-based study. Epidemiol Health. 2012;34

89. Esmaillzadeh A, Mirmiran P, Azadbakht L, Etemadi A, Azizi F. High prevalence of the metabolic syndrome in Iranian adolescents. Obesity (Silver Spring). 2006;14(3):377-82. doi: 10.1038| oby.2006.50. [PubMed: 16648607]

90. Kelishadi R, Gheiratmand R, Ardalan G, Adeli K, Mehdi Gouya M Mohammad Razaghi E, et al. Association of anthropometric indices with cardiovascular disease risk factors among children and adolescents: CASPIAN Study. Int J Cardiol. 2007;117(3):340-8. doi: 10.1016/j.ijcard.2006.06.012. [PubMed:16860411]

91. Kelishadi R, Gouya MM, Adeli K, Ardalan G, Gheiratmand R, Majdzadeh R, et al. Factors associated with the metabolic syndrome in a national sample of youths: CASPIAN Study. Nutr Metab Cardiovasc Dis. 2008;18(7):461-70. doi:10.1016/j.numecd.2007.02.014 [PubMed: 17935959]

92. Kelishadi R, Poursafa P, Keramatian K. Overweight, air and noise pollution: Universal risk factors for pediatric pre-hypertension. $J$ Res Med Sci. 2011;16(9):1234-50. [PubMed: 22973395]

93. Sepanlou SG, Kamangar F, Poustchi H, Malekzadeh R. Reducing the burden of chronic diseases: a neglected agenda in Iranian health care system, requiring a plan for action. Arch Iran Med. 2010;13(4):340-50. [PubMed: 20597569]

94. Beaglehole R, Bonita R, Alleyne G, Horton R, Li L, Lincoln P, et al. UN High-Level Meeting on Non-Communicable Diseases: addressing four questions. Lancet. 2011;378(9789):449-55. [PubMed: 21665266] 